\title{
Brimonidine in the treatment of glaucoma and ocular hypertension
}

\author{
Louis B Cantor \\ Department of Ophthalmology, \\ Indiana University, Indianapolis, IN, \\ USA
}

\begin{abstract}
Treatment in glaucoma aims to lower intraocular pressure (IOP) to reduce the risk of progression and vision loss. The alpha2-adrenergic receptor agonist brimonidine effectively lowers IOP and is useful as monotherapy, adjunctive therapy, and replacement therapy in open-angle glaucoma and ocular hypertension. A fixed combination of brimonidine and timolol, available in some countries, reduces IOP as effectively as concomitant therapy with brimonidine and timolol and offers the convenience of 2 drugs in a single eyedrop. Brimonidine is safe and well tolerated. Its most common side-effects are conjunctival hyperemia, allergic conjunctivitis, and ocular pruritus. The newest formulation of brimonidine, brimonidine-Purite $0.1 \%$, has a higher $\mathrm{pH}$ to improve the ocular bioavailability of brimonidine. This formulation contains the lowest effective concentration of brimonidine and is preserved with Purite ${ }^{\mathbb{B}}$ to enhance ocular tolerability. Brimonidine-Purite $0.1 \%$ is as effective in reducing IOP as the original brimonidine $0.2 \%$ solution preserved with benzalkonium chloride. Recent results from preclinical and clinical studies suggest that brimonidine may protect retinal ganglion cells and their projections from damage and death independently of its effects on IOP. The potential for neuroprotection with brimonidine is an added benefit of its use in glaucoma and ocular hypertension.
\end{abstract}

Keywords: brimonidine, preservative, glaucoma, intraocular pressure, neuroprotection

\section{Introduction}

Glaucoma is an optic neuropathy characterized by acquired loss of retinal ganglion cells (RGCs) and atrophy of the optic nerve leading to vision loss. Elevated intraocular pressure (IOP) is a primary risk factor both for the development of glaucoma and for progression of optic nerve changes and visual field loss in the disease. Abundant evidence indicates that elevated IOP can cause the neuropathology of glaucoma. Clinical experience with angle-closure glaucoma and numerous preclinical studies in rats and primates have shown that acute and sustained increases in IOP can cause optic nerve damage (Morrison 2005; Rasmussen and Kaufman 2005). Primary openangle glaucoma (POAG), the most common type of glaucoma in white populations, is characterized by chronically elevated IOP with no known cause for the elevated IOP or optic neuropathy. But many individuals with elevated IOP do not show signs of glaucomatous optic nerve damage, and conversely, many individuals with IOP consistently within the normal range (less than $21 \mathrm{mmHg}$ ) have glaucoma (Klein et al 1992). These findings suggest that factors beyond IOP have a role in the etiology of the disease (Drance 1997).

\section{IOP-lowering treatment}

Regardless of the etiology of the disease, at present, the aim of treatment in glaucoma is to reduce IOP. Recent randomized, controlled clinical trials have shown that lowering IOP is effective in delaying or preventing the development of glaucoma in 
patients with ocular hypertension (OHT) and in delaying or halting the progression of established glaucoma (Heijl et al 2002; Kass et al 2002). IOP reduction is beneficial in reducing the risk of progression of vision loss even when IOP is already within the normal range (Collaborative Normal-Tension Glaucoma Study Group 1998). Evidence suggests that very low IOP provides the best visual outcomes for patients (The AGIS Investigators 2000; Lichter et al 2001). Analysis of data from the Early Manifest Glaucoma Trial showed a $10 \%$ reduction in the risk of progression associated with each $1 \mathrm{mmHg}$ of IOP reduction (Leske et al 2003).

IOP-lowering drugs are currently the only medical treatment approved for glaucoma management. The classes of ocular hypotensive drugs commonly used to reduce IOP in glaucoma and OHT include prostaglandin analogues, beta-adrenergic receptor antagonists, alpha-adrenergic receptor agonists, carbonic anhydrase inhibitors, and parasympathomimetics. The once-daily prostaglandin analogues (bimatoprost, latanoprost, travoprost) reduce IOP most effectively (Hedman and Alm 2000; Netland et al 2001; Higginbotham et al 2002) and are often used as initial monotherapy. Not all patients can use prostaglandin analogues, however. Further, for many patients the IOP lowering obtained with monotherapy is inadequate. Even patients with OHT or early glaucoma are likely to need more than 1 medication to reach sufficiently low pressures. For example, in the Ocular Hypertension Treatment Study (OHTS) by year 5 almost $40 \%$ of patients needed 2 or more medications to achieve their target IOP (Kass et al 2002), and in the Collaborative Initial Glaucoma Treatment Study (CIGTS) after year 2 more than 75\% of patients needed 2 or more medications to reach their target IOP (Lichter et al 2001).

Brimonidine, the only selective alpha-adrenergic receptor agonist approved for chronic treatment in glaucoma, is indicated for reducing IOP in patients with open-angle glaucoma or OHT. Brimonidine is contraindicated in patients receiving monoamine oxidase inhibitor therapy, because antidepressants decrease the metabolism of circulating monoamines, leading to an increase in levels of endogenous monoamines that might inhibit the IOP-lowering effect of brimonidine. Brimonidine is also contraindicated in patients with hypersensitivity to any component of the medication, and it should not be used in children under the age of 2 because there have been reports of apnea, bradycardia, hypothermia, hypotonia, lethargy, and unresponsiveness in infants receiving brimonidine treatment (Berlin et al 2001; Prok and Hall 2003).

\section{Pharmacology and mechanism of action of brimonidine}

Brimonidine is a selective alpha2-adrenergic receptor agonist that shows up to 1780-fold selectivity for alpha2over alpha1-adrenergic receptors (Cantor 2000). After topical instillation, brimonidine reduces IOP within 1 hour, and the peak effect occurs at 2-3 hours after dosing (Walters 1996). The trough effect occurs at 10-14 hours after dosing. Brimonidine is usually dosed twice daily, and no additional IOP lowering is provided at morning trough with tid versus bid dosing (Walters 1996). Brimonidine has a dual mechanism of IOP lowering: it both reduces aqueous humor production and stimulates aqueous humor outflow through the uveoscleral pathway (Toris et al 1995). The predominant effect of short-term brimonidine treatment is inhibition of aqueous production, whereas the predominant effect of chronic treatment is stimulation of aqueous humor outflow through the uveoscleral pathway (Toris et al 1999).

\section{Pharmacokinetics of topical brimonidine}

Pharmacokinetic studies in rabbits and monkeys have shown that topical brimonidine readily penetrates the eye and reaches pharmacologically active concentrations in the aqueous humor and ciliary body, the putative sites of its IOP-lowering activity (Acheampong et al 1995, 2002). The primary absorption route for brimonidine is via the cornea (Cantor 2000). Brimonidine that reaches the systemic circulation after topical administration in humans is rapidly metabolized and has a short plasma half-life of approximately 2 hours (Cantor 2000). The rapid metabolism and systemic clearance of brimonidine minimizes potential systemic effects of the drug, and twice- or thrice-daily dosing of brimonidine $0.2 \%$ is not associated with clinically significant cardiovascular or pulmonary systemic effects in adults (Cantor 2000).

Pharmacologically active concentrations of brimonidine are found in vitreous humor samples following topical administration of brimonidine $0.2 \%$ in rats, rabbits, monkeys, and humans (Kent et al 2001; Acheampong et al 2002). This is important because brimonidine may be present at the retina in concentrations sufficient for direct effects on RGCs. 


\section{Potential for neuroprotection}

As it has become recognized that glaucoma is a multifactorial, progressive neuropathy that often occurs independently of elevated IOP, the diagnosis of glaucoma has changed from one based on IOP to one based on the optic nerve and visual field (Weinreb and Levin 1999). This paradigm shift has prompted investigation of a new approach to therapy in glaucoma called neuroprotection. The goal of neuroprotection is to slow or prevent death of neurons and maintain their physiological function (Weinreb and Levin 1999). One important advantage of a neuroprotective strategy is that treatment is possible even when the etiology of the disease is unknown or differs among patients (Weinreb and Levin 1999). A neuroprotective treatment in glaucoma might have no effect on IOP, but it would promote the survival of RGCs and their axons (the optic nerve fibers), and it could be effective regardless of the specific etiology of the disease (Weinreb and Levin 1999).

Neuroprotection has been investigated as a therapeutic approach for neurodegenerative conditions including stroke, spinal cord injury, Parkinson's disease, Huntington's disease, amyotrophic lateral sclerosis, and Alzheimer's disease. It may be difficult to achieve neuroprotection in acute conditions such as stroke, because treatment would probably have to begin at the time of the insult or soon after to prevent irreversible neuronal loss (Osborne et al 2004), but neuroprotection may be easier to achieve in chronic diseases characterized by progressive cell loss, such as open-angle glaucoma.

Preclinical studies have shown that brimonidine has neuroprotective effects in animal models of optic nerve injury relevant to glaucoma including partial optic nerve crush, chronic ocular hypertension induced by laser cautery of episcleral and limbal veins, and retinal ischemia induced either by transient elevation of IOP or ligature of ophthalmic vessels (Yoles et al 1999; Donello et al 2001; WoldeMussie et al 2001; Mayor-Torroglosa et al 2005). Brimonidine was shown to promote RGC survival in each of these models, and in most studies protection of visual function was also demonstrated through measurements of the compound action potential or the ERG b-wave. The effects of brimonidine are evident after topical administration of a $0.1 \%$ or $0.5 \%$ solution of drug (Vidal-Sanz et al 2001) and are mediated by activation of alpha2-adrenergic receptors (Donello et al 2001). Moreover, the effects appear to be independent of IOP lowering, because systemic administration of brimonidine, which does not reduce IOP, is also neuroprotective (Yoles et al 1999). Recent clinical studies of brimonidine, discussed later in this review, have suggested that topical brimonidine treatment may also protect RGCs in human glaucoma.

\section{Brimonidine formulations}

The original brimonidine $0.2 \%$ formulation (Alphagan ${ }^{\circledR}$, Allergan, Inc, Irvine, CA, USA) has a $\mathrm{pH}$ of 6.4 and is preserved with benzalkonium chloride (BAK). BAK is the antimicrobial preservative most commonly used in ophthalmic solutions, but chronic exposure to solutions containing high concentrations of BAK has been associated with harmful effects on the corneal surface (Noecker 2001; Noecker et al 2004). Moreover, chronic treatment of glaucoma and OHT patients with IOP-lowering ophthalmic solutions preserved with BAK has been reported to result in subclinical inflammation evident by increased expression of HLA-DR on conjunctival epithelial cells (Cvenkel and Ihan 2002). This is a clinical concern, because chronic inflammation and fibrosis can decrease the success rate of trabeculectomy surgery (Skuta and Parrish 1987).

Brimonidine has been reformulated to improve its tolerability while maintaining its ocular bioavailability and IOP-lowering efficacy. The newer formulations of brimonidine are preserved with Purite ${ }^{\circledR}$, a stabilized oxychloro complex and oxidative preservative that is converted to natural tear components (sodium and chloride ions, oxygen, and water) when exposed to light (Katz 2002). Purite is a microbicide and is non-toxic to mammalian cells (Grant et al 1996). The first reformulation of brimonidine that was introduced contains brimonidine $0.15 \%$ in a buffered solution of $\mathrm{pH} 7.2$ preserved with Purite $0.005 \%$ (Alphagan ${ }^{\circledR}$ P $0.15 \%$, Allergan Inc, Irvine, CA, USA). Although this formulation has a reduced concentration of brimonidine, it was shown in clinical trials to have the same IOP-lowering efficacy and better tolerability compared with the original brimonidine $0.2 \%$ formulation (Katz 2002) because the increase in $\mathrm{pH}$ provided better bioavailability (Dong et al 2004). More recently, a $0.1 \%$ formulation of brimonidine preserved with Purite at a $\mathrm{pH}$ of 7.7 was introduced (Alphagan ${ }^{\circledR}$ P $0.1 \%$, Allergan Inc, Irvine, CA, USA). As discussed in detail below, the new brimonidinePurite $0.1 \%$ formulation also shows efficacy equivalent to the original brimonidine $0.2 \%$ formulation. Animal studies have shown that aqueous humor levels of drug are the same with the newer formulations preserved with Purite and the old formulation preserved with BAK, despite the lower concentration of drug in the bottle, because at higher $\mathrm{pH}$ more brimonidine is non-ionized, and brimonidine is more 
readily absorbed into the eye (Dong 2004; Allergan, data on file).

\section{Clinical efficacy of brimonidine in reducing IOP}

In its 1-year pivotal trials for drug approval, twice-daily brimonidine $0.2 \%$ reduced IOP as well as or better than timolol at peak effect ( 2 hours after dosing) but less effectively than timolol at morning trough (Schuman et al 1997; LeBlanc 1998; Katz 1999). The efficacy of brimonidine was sustained over long-term use, and after four years of treatment, brimonidine and timolol provided comparable IOP lowering at both peak and trough effect (David 2001). Brimonidine was well tolerated in the pivotal trials. Common side-effects of treatment included oral dryness, ocular hyperemia, and ocular allergy. The 1-year incidence of treatment-related ocular allergy to brimonidine was $11.5 \%$ (Katz 1999), but this incidence may have been overestimated because of the confusion of dry eye, seasonal allergic conjunctivitis, or bacterial conjunctivitis with drugrelated ocular allergy (Melamed and David 2000).

Brimonidine has been compared with dorzolamide as monotherapy in glaucoma and OHT in 3 separate randomized, double-masked studies with a crossover design (Stewart et al 2000; Sharpe et al 2004; Whitson et al 2004). In each of these studies, brimonidine and dorzolamide showed comparable efficacy at trough effect, but at peak effect at 2 hours after dosing, brimonidine reduced IOP by $0.7-1.4 \mathrm{mmHg}$ more than dorzolamide. There was no overall difference between drugs in the frequency of side-effects, but ocular stinging and burning were more often associated with dorzolamide treatment.

The versatility of brimonidine in reducing IOP was demonstrated in a large, open-label study involving 2335 patients. In this study, brimonidine effectively reduced IOP whether used as monotherapy, replacement therapy, or adjunctive therapy (Lee et al 2000). As adjunctive therapy, brimonidine provided significant mean additional IOP lowering when added to other ocular hypotensive medications including beta-blockers, carbonic anhydrase inhibitors, and the prostaglandin analogue latanoprost (Lee and Gornbein 2001). Several randomized controlled clinical studies in patients with glaucoma or OHT subsequently confirmed that brimonidine provides significant additional mean decreases in IOP when added to ongoing beta-blocker therapy (Simmons 2001; Simmons and Earl 2002; Sall et al 2003; Solish et al 2004). Other randomized controlled trials showed that brimonidine effectively reduces IOP when used adjunctively with a prostaglandin analogue (bimatoprost or latanoprost) (Netland et al 2003; Zabriskie and Netland 2003; Konstas et al 2005).

Brimonidine has been demonstrated to be more effective than dorzolamide when used as adjunctive therapy with a beta-blocker and at least as effective as dorzolamide when used as adjunctive therapy with latanoprost. In 2 randomized controlled trials that compared the efficacy and safety of brimonidine and dorzolamide as adjunctive therapy with beta-blockers, the reduction from baseline IOP (measured at peak effect) was significantly greater with adjunctive brimonidine than with adjunctive dorzolamide (Simmons 2001; Carrasco Font et al 2004). Brimonidine-Purite 0.15\% was compared with dorzolamide as adjunctive therapy with latanoprost in a randomized, double-masked, crossover trial in 33 glaucoma patients who had uncontrolled IOP after at least a 3-week run-in on latanoprost monotherapy (Konstas et al 2005). Each study drug was given twice daily as adjunctive therapy with latanoprost for 6 weeks, with a 6week washout between treatment periods. The primary outcome measure was circadian IOP, measured at 7 timepoints over 24 hours after 6 weeks of adjunctive therapy. The between-group differences in mean IOP reduction from baseline were not statistically significant. Of the 31 enrolled patients who had data available for analysis, 1 (3.2\%) had the same circadian IOP (average of all 7 measurements) on both drugs, 19 (61.3\%) had lower circadian IOP with brimonidine-Purite, and 11 (35.5\%) had lower circadian IOP with dorzolamide, suggesting that brimonidine-Purite $0.15 \%$ is at least as effective as dorzolamide in providing 24-hour IOP control when added to latanoprost.

\section{Clinical comparison of brimonidine-Purite $0.1 \%$ and brimonidine $0.2 \%$}

A prospective, randomized, double-masked, parallel-group clinical trial compared brimonidine-Purite $0.1 \%$ with brimonidine $0.2 \%$ for IOP-lowering efficacy and tolerability in patients with glaucoma or OHT (Allergan, data on file). The study was carried out at 27 centers across the United States. Patients with glaucoma or OHT in each eye were randomized to treatment with either brimonidine-Purite $0.1 \%(\mathrm{n}=215)$ or brimonidine $0.2 \%(\mathrm{n}=218)$ thrice daily for 12 months. Follow-up visits were scheduled at weeks 2 and 6 and months 3, 6, 9, and 12. IOP was measured at $8 \mathrm{AM}$ (trough effect, immediately prior to the morning dose), 10 AM (morning peak effect), and 4 PM (afternoon peak effect, 
2 hours after the afternoon dose) at all follow-up study visits except month 9 , when it was measured at $8 \mathrm{AM}$ and $10 \mathrm{AM}$ only. The primary efficacy measure was mean IOP in the intent-to-treat patient population (all randomized patients) with last observation carried forward for missing values. All patients were treated bilaterally, and the average IOP from both eyes was used in the analyses.

Baseline demographic and ophthalmic characteristics of patients were similar between the 2 treatment groups. Mean IOP at baseline was also comparable between the 2 treatment groups at each hour. Throughout follow-up, mean IOP in each treatment group ranged from 17 to $22 \mathrm{mmHg}$ and was significantly lower than at baseline $(\mathrm{p}<0.001)$. The absolute values of the limits of the $95 \%$ confidence interval (CI) of the between-group difference in mean IOP were $<1.0 \mathrm{mmHg}$ at 12 of 17 timepoints and $<1.5 \mathrm{mmHg}$ at all 17 timepoints, demonstrating equivalent efficacy of the study formulations (Figure 1). Analysis of mean change from baseline IOP also showed equivalent efficacy of the study formulations, with the absolute values of the limits of the $95 \%$ CI of the between-group difference $<1.0 \mathrm{mmHg}$ at 9 of 17 timepoints and consistently $<1.5 \mathrm{mmHg}$. The only significant differences in mean IOP reduction between treatment groups were at $4 \mathrm{PM}$ at months 3 and 12, when the mean IOP reduction was significantly greater with brimonidine-Purite $0.1 \%$ than with brimonidine $0.2 \%(\mathrm{p} \leq 0.043)$. BrimonidinePurite $0.1 \%$ provided sustained IOP lowering over 12 months of treatment and was as effective as brimonidine $0.2 \%$ in reducing IOP at all timepoints. Figure 2 shows the mean change from baseline IOP with each formulation at the 10 AM timepoint of peak effect.

The percentage of patients with 1 or more treatmentrelated adverse events was lower in the brimonidine-Purite $0.1 \%$ group $(41.4 \%)$ than in the brimonidine $0.2 \%$ group $(53.2 \%, \mathrm{p}=0.014)$. The only individual treatment-related adverse event with a significant difference in incidence between treatment groups was oral dryness, which was less frequent in the brimonidine-Purite $0.1 \%$ group $(1.4 \%$ of patients) than in the brimonidine $0.2 \%$ group $(5.5 \%$ of patients, $\mathrm{p}=0.019$ ). Biomicroscopic findings of increased severity of lid erythema and lid edema were also less common in the brimonidine-Purite $0.1 \%$ group $(\mathrm{p}=0.028$ and $\mathrm{p}=0.006$, respectively).

The rate of discontinuations for adverse events was significantly lower in the brimonidine-Purite $0.1 \%$ group $(21.4 \%)$ than in the brimonidine $0.2 \%$ group $(33.5 \%$, $\mathrm{p}=0.005$ ). Only 1 patient in the brimonidine-Purite $0.1 \%$ group discontinued for a non-ocular, treatment-related adverse event (oral dryness). In contrast, patients in the brimonidine $0.2 \%$ group discontinued for several non-ocular treatment-related adverse events including asthenia, hypotension, somnolence, depression, and insomnia, as well as oral dryness.

In summary, the results of this trial showed that brimonidine-Purite $0.1 \%$ is statistically equivalent to

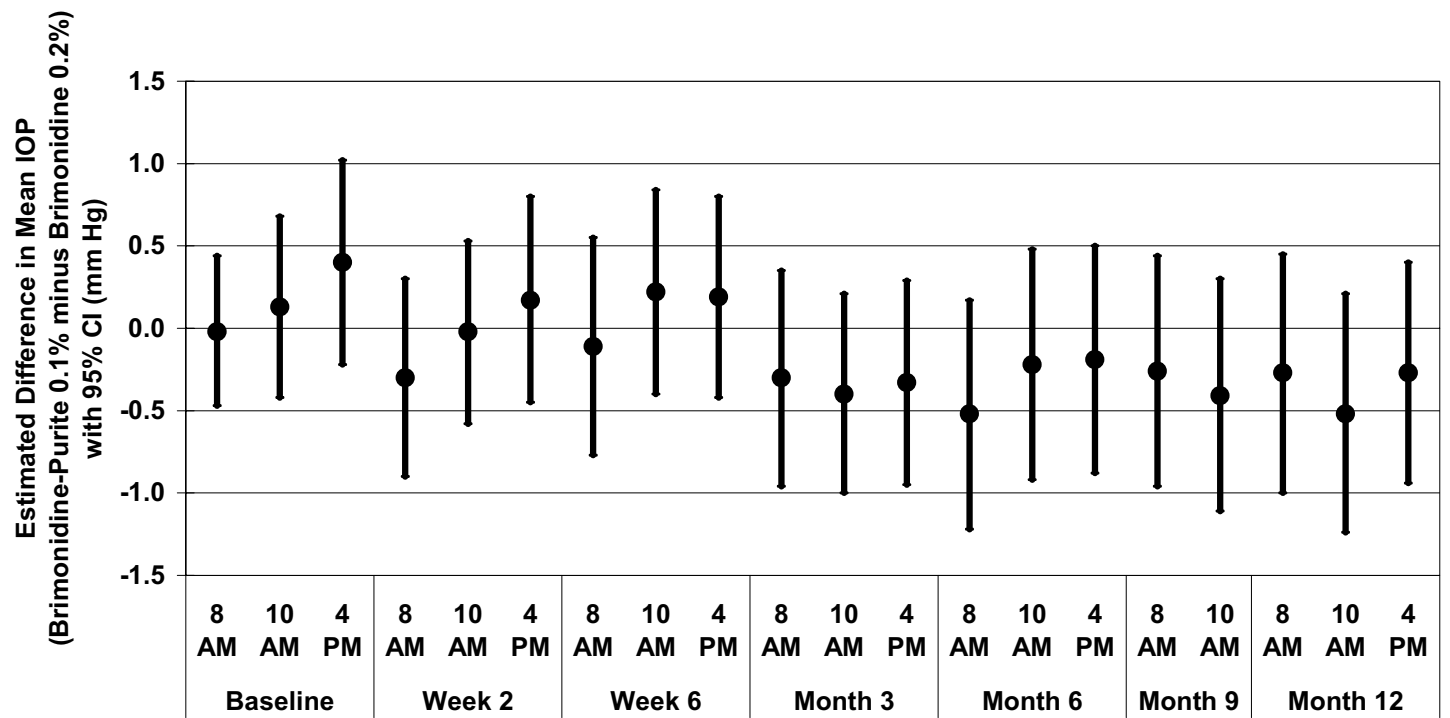

Figure I Equivalent IOP-lowering efficacy of brimonidine-Purite $0.1 \%$ and brimonidine $0.2 \%$. In a I-year clinical comparison study of the 2 formulations, the $95 \% \mathrm{Cl}$ of the difference in mean IOP between treatment groups (brimonidine-Purite $0.1 \%$ minus brimonidine $0.2 \%$ ) was consistently within the range of $-\mathrm{I} .5 \mathrm{mmHg}$ to $1.5 \mathrm{mmHg}$, demonstrating equivalent efficacy of the study formulations.

Abbreviations: $\mathrm{Cl}$, confidence interval; IOP, intraocular pressure. 


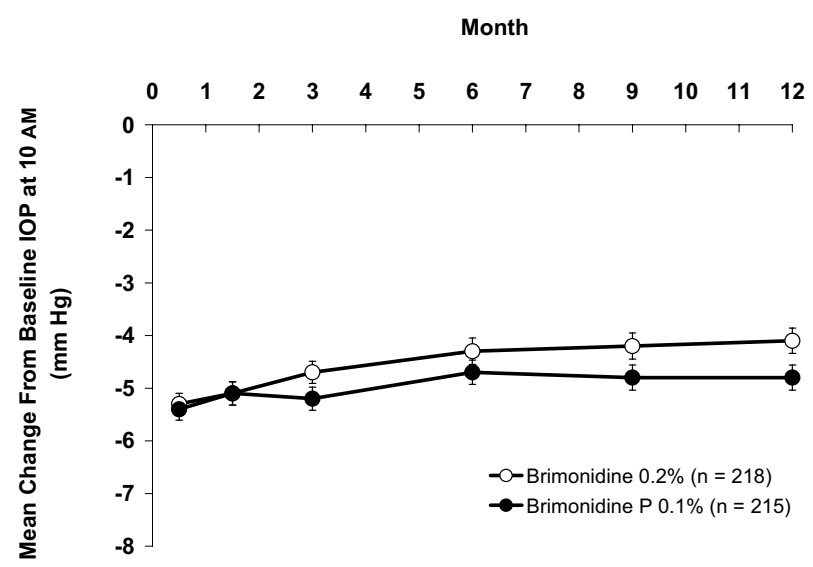

Figure 2 Mean change from baseline IOP. Both brimonidine-Purite $0.1 \%$ and brimonidine $0.2 \%$ provided significant IOP reductions that were sustained throughout I year of therapy. The mean IOP reduction was equivalent with the 2 formulations throughout follow-up.

Abbreviations: IOP, intraocular pressure.

brimonidine $0.2 \%$ in IOP-lowering efficacy and at least as well tolerated in patients with glaucoma and OHT.

\section{Safety and tolerability}

Brimonidine has a favorable safety and tolerability profile. Unlike the beta-adrenergic antagonists, there are no cardiopulmonary contraindications to its use, and brimonidine can be safely and effectively used by patients on systemic anti-hypertensive beta-blocker therapy (Schuman 2000). In contrast to the prostaglandin analogues, brimonidine has not been associated with eyelash growth or increased pigmentation of the iris or eyelids. The sideeffects associated with brimonidine treatment are usually ocular and include conjunctival hyperemia, allergic conjunctivitis, and ocular pruritus. The most common systemic side-effects are oral dryness and fatigue or drowsiness.

The reduced drug concentration in the brimonidinePurite $0.15 \%$ formulation compared with brimonidine $0.2 \%$ led to an improvement in tolerability (Katz 2002). In a randomized, double-masked, comparison trial of these formulations, glaucoma and OHT patients treated with the brimonidine-Purite $0.15 \%$ formulation had a $41 \%$ lower incidence of allergic conjunctivitis (Katz 2002). Oral dryness, conjunctival hyperemia, and eye discharge were also significantly less common with brimonidine-Purite $0.15 \%$ than with brimonidine $0.2 \%$ (Katz 2002).

The brimonidine-Purite $0.1 \%$ formulation also has a favorable safety and tolerability profile. Because the active drug concentration is $33 \%$ less than in the brimonidinePurite $0.15 \%$ formulation, the ocular surface is exposed to less drug, and systemic absorption of brimonidine through the nasolacrimal pathway is likely to be further reduced with the $0.1 \%$ formulation. Consistent with this suggestion, in a study in rabbits, aqueous humor levels of drug were similar after dosing with either the brimonidine-Purite $0.15 \%$ or $0.1 \%$ formulation, but plasma levels of brimonidine were lower in animals dosed with the brimonidine-Purite $0.1 \%$ formulation (Allergan, data on file). These results suggest the possibility that the new brimonidine-Purite $0.1 \%$ formulation may have an improved systemic safety profile over brimonidine-Purite $0.15 \%$, but clinical comparison studies will be needed to confirm this possibility.

\section{Patient acceptance and compliance with brimonidine treatment}

Brimonidine treatment is usually well received by patients. In the phase 3 clinical comparison of brimonidine-Purite $0.15 \%$ and brimonidine $0.2 \%$, most patients (approximately $80 \%$ ) considered their eyedrops to be comfortable and were satisfied with their treatment (Katz 2002). Patient comfort ratings and satisfaction ratings were significantly higher with the brimonidine-Purite $0.15 \%$ formulation than with brimonidine $0.2 \%$.

It is generally recognized that lack of compliance with medical treatment is a significant problem in many diseases, and compliance may be particularly poor in slowly progressive, largely asymptomatic chronic diseases such as glaucoma. Poor compliance can lead to treatment failure, yet up to $80 \%$ of glaucoma patients may not take their medication as prescribed (Olthoff et al 2005). The reasons for non-compliance in glaucoma are not completely understood, but patients often mention forgetfulness as their main reason for not taking their eyedrops (Taylor et al 2002), and self-reported difficulty in remembering to take glaucoma medications has been significantly associated with patient nonadherence to treatment (Sleath et al 2006). Other reasons for non-compliance with medical treatment in glaucoma may include cost of medications, inconvenience of the dosing schedule, inability to instill the eyedrops correctly, and lack of understanding of the need for chronic therapy (Winfield et al 1990). Side-effects are usually not a significant cause of noncompliance (Olthoff et al 2005; Taylor et al 2002). Required instillation of drops at 3 or more times during the day is strongly associated with reduced compliance (Olthoff et al 2005), but this is generally not a concern with brimonidine treatment, because brimonidine is usually dosed 
twice daily. There is currently no evidence to suggest that compliance with brimonidine treatment is either better or worse than treatment compliance with other classes of ocular hypotensive medications.

\section{Clinical trials of neuroprotection with brimonidine}

An initial trial evaluating potential neuroprotection by brimonidine in patients with acute angle-closure glaucoma found no difference between the number of patients who progressed during treatment with brimonidine or timolol (Aung et al 2004). The study was limited because overall, only 59 patients completed the study, and only 11 of these patients progressed during the study, but there was a trend for the rate of progression to be lower in patients treated with brimonidine (Aung et al 2004). A second trial evaluated potential neuroprotection by brimonidine in patients with non-arteritic anterior ischemic optic neuropathy (NAION) (Wilhelm et al 2006). This was a randomized, doublemasked study that evaluated 29 patients with first eye involvement who were treated with brimonidine $0.2 \%$ or placebo within the first week after loss of visual acuity. After 3 months of treatment there was a trend for better visual field results on all measures in the brimonidine group. The study was stopped after an interim analysis, however, when the investigators concluded that the number of patients needed for statistical significance could not be recruited within a practical time frame.

A randomized, double-masked, pilot study evaluated potential neuroprotection by brimonidine in eyes of patients undergoing laser treatment for extrafoveal or juxtafoveal choroidal neovascularization (CNV) (Ferencz et al 2005). Eyes were treated with brimonidine $0.2 \%$ (study group, 11 eyes) or placebo (control group, 9 eyes) twice daily, beginning at $4-48$ hours before laser treatment and continuing afterwards for 1 month. In each treatment group, 2 eyes had recurrence of $\mathrm{CNV}$ in the subfoveal region and severe visual loss after the photocoagulation procedure. For the remaining 16 eyes, mean visual acuity at 2 months after laser treatment was improved in the brimonidine group but not in the control group. The investigators suggested that the improvement in visual acuity in brimonidine-treated patients most likely resulted from an ability of brimonidine to protect RGCs from damage induced by noxious substances released from cells destroyed by the laser treatment (Ferencz et al 2005).
A recent trial has provided encouraging results suggesting neuroprotective effects of brimonidine in glaucoma patients (Tsai and Chang 2005). In this prospective, randomized, unmasked study, 78 patients with newly diagnosed POAG were treated with brimonidine $0.2 \%$ twice daily or timolol $0.5 \%$ ophthalmic gel-forming solution once daily in the morning for 12 months. Eligible patients were required to have untreated IOP $>21 \mathrm{mmHg}$, a glaucomatous appearance of the optic disc, and an abnormal visual field on standard automated perimetry. IOP was measured at 2-month intervals throughout the study. Retinal nerve fiber layer (RNFL) thickness was measured using scanning laser polarimetry (GDx) at baseline and at the end of the study.

At baseline, there were no significant between-group differences in patient age, sex, visual field mean deviation and corrected pattern standard deviation, and IOP. Baseline RNFL thickness measurements including the ellipse average, superior average, temporal average, inferior average, and nasal average were also comparable between treatment groups. After 12 months of follow-up, patients in the timolol group showed a significant mean decrease in all RNFL measurements ( $p \leq 0.044)$ consistent with glaucomatous progression of RGC loss. In contrast, patients in the brimonidine group showed no significant changes in any of the RNFL thickness measurements ( $\mathrm{p} \geq 0.14)$ and there was no evidence for glaucomatous loss of the RNFL. The between-group comparison of mean change from baseline RNFL thickness showed significantly greater loss of temporal average $(\mathrm{p}=0.005)$, inferior average $(\mathrm{p}=0.016)$, and ellipse average $(\mathrm{p}=0.020)$ RNFL thickness in the timolol group compared with the brimonidine group.

No significant between-group differences in mean IOP were found at baseline or at any follow-up study visit ( $\mathrm{p}$ $\geq 0.038$ ). The mean IOP reduction at the month 12 visit was $5.6 \mathrm{mmHg}$ in the brimonidine group and $5.3 \mathrm{mmHg}$ in the timolol group $(p=0.16)$. Therefore, the differences in RNFL loss between the treatment groups are unlikely to be explained by better IOP control in the brimonidine group. Instead, the findings are likely to be explained by a direct neuroprotective effect of brimonidine, which promoted the survival of RGCs and their axons, and thereby prevented glaucomatous damage to the RNFL. The brimonidine-Purite $0.15 \%$ and $0.1 \%$ formulations would be expected to have a similar neuroprotective effect, if (as is likely) neuroprotection results from brimonidine activity at the retina, rather than from systemic effects of the drug. 
This latest study provides the strongest published results to date suggesting that neuroprotection with brimonidine may be a viable strategy for treatment in glaucoma. The use of scanning laser polarimetry allowed quantitative evaluation of structural changes in the RNFL and demonstrated possible neuroprotection of RGCs by brimonidine. Further studies that evaluate the ability of brimonidine and other potential neuroprotective agents to prevent the progression of functional changes and visual field loss in glaucoma are eagerly anticipated.

\section{Fixed combination of brimonidine and timolol}

A fixed combination of brimonidine $0.2 \% /$ timolol $0.5 \%$ (Combigan ${ }^{\circledR}$, Allergan Inc, Irvine, CA, USA) is available in some countries. The fixed combination is preserved with BAK and contains $0.2 \%$ brimonidine and timolol $0.5 \%$. A 3-month, randomized, double-masked, clinical study in 1159 patients with glaucoma or OHT showed that the brimonidine/timolol fixed combination is more efficacious than either of its component medications (Craven et al 2005). In this study, at all follow-up timepoints the reduction from baseline IOP was significantly greater in patients treated with the brimonidine/timolol fixed combination BID than in those treated with timolol BID monotherapy ( $p \leq 0.026$ ). At all $8 \mathrm{AM}, 10 \mathrm{AM}$, and $3 \mathrm{AM}$ timepoints, the mean reduction from baseline IOP was also greater for patients treated with the fixed combination than for those treated with brimonidine tid monotherapy $(\mathrm{p}<0.001)$ (Craven et al 2005). The fixed combination bid was better tolerated than brimonidine tid monotherapy but not as well tolerated as timolol bid monotherapy.

The brimonidine/timolol fixed combination lowers IOP as effectively as concomitant therapy with separate drops of brimonidine $0.2 \%$ and timolol $0.5 \%$ (Gõni 2005). In a randomized, double-masked, clinical trial, 371 glaucoma or OHT patients with inadequate IOP control after at least 3 weeks of run-in monotherapy were switched to either the fixed combination of brimonidine/timolol bid or concomitant timolol bid and brimonidine bid therapy (Gõni 2005). Both treatments provided significant additional IOP lowering from baseline on monotherapy. Differences between the fixed combination and concomitant therapy were $\leq 0.35 \mathrm{mmHg}$ for mean IOP and $\leq 0.30 \mathrm{mmHg}$ for mean change from baseline IOP at all timepoints over the 3-month study, and none of the differences were statistically significant. No differences in tolerability were noted between the fixed combination and concomitant therapy. Use of the fixed combination rather than separate drops of brimonidine and timolol does, however, reduce corneal exposure to BAK, and this may help reduce corneal toxicity associated with chronic treatment (Gõni 2005). Dosing of the fixed combination is also easier and more convenient, because there is no need to wait between instillation of separate drops. It was suggested that this increase in convenience may result in better patient compliance and a higher rate of treatment success (Choudhri et al 2000).

\section{Place of brimonidine in therapy for glaucoma and OHT}

Brimonidine can be used as monotherapy in glaucoma and OHT, but it is more commonly used as adjunctive therapy. The once-daily prostaglandin analogue drugs are generally the preferred choice for first-line therapy because they provide the lowest IOP and consistent IOP control throughout the day. Beta-blockers are also considered an appropriate choice of first-line therapy for some patients. Regardless of the first-line therapy, most patients eventually require more than 1 ocular hypotensive medication for adequate IOP control, and brimonidine is considered a preferred choice for adjunctive therapy because of its effectiveness in adjunctive therapy with multiple classes of IOP-lowering medications and its favorable safety and tolerability profile (Lee and Higginbotham 2005). There is particularly strong evidence that brimonidine is effective when used in combination with a beta-blocker, either when given as a separate drop or in the brimonidine/timolol fixed combination. For patients whose treatment regimen includes both brimonidine and timolol, use of the fixed combination may be preferred because fixed-combination treatment offers potential advantages of increased compliance, reduced costs, and reduced cumulative exposure to BAK (Fechtner and Realini 2004).

The brimonidine-Purite $0.15 \%$ formulation is clearly preferable to the brimonidine $0.2 \%$ formulation because it provides the same efficacy and better tolerability. The brimonidine-Purite $0.1 \%$ formulation also provides the same efficacy as brimonidine $0.2 \%$, and the reduced drug exposure associated with its use is likely to result in improved longterm safety and tolerability of treatment. Because brimonidine-Purite $0.15 \%$ and the new brimonidine-Purite $0.1 \%$ are formulated at a different $\mathrm{pH}$, however, comparative clinical studies will be needed to determine their comparative efficacy and safety. 
Results of the clinical trials of neuroprotection with brimonidine are promising, and it now seems reasonable to consider the potential for neuroprotection when choosing therapy for glaucoma. Brimonidine can be used safely and reliably to reduce IOP in glaucoma and OHT, and it may also reduce the risk of glaucomatous progression independent of its effects on IOP.

\section{References}

Collaborative Normal-Tension Glaucoma Study Group. 1998. Comparison of glaucomatous progression between untreated patients with normaltension glaucoma and patients with therapeutically reduced intraocular pressures. Am J Ophthalmol, 126:487-97.

The AGIS Investigators. 2000. The Advanced Glaucoma Intervention Study (AGIS): 7. The relationship between control of intraocular pressure and visual field deterioration. Am J Ophthalmol, 130:429-40.

Acheampong AA, Shackleton M, John B, et al. 2002. Distribution of brimonidine into anterior and posterior tissues of monkey, rabbit, and rat eyes. Drug Metab Dispos, 30:421-9.

Acheampong AA, Shackleton M, Tang-Liu DD. 1995. Comparative ocular pharmacokinetics of brimonidine after a single dose application to the eyes of albino and pigmented rabbits. Drug Metab Dispos, 23:708-12.

Aung T, Oen FT, Wong HT, et al. 2004. Randomised controlled trial comparing the effect of brimonidine and timolol on visual field loss after acute primary angle closure. Br J Ophthalmol, 88:88-94.

Berlin RJ, Lee UT, Samples JR, et al. 2001. Ophthalmic drops causing coma in an infant. $J$ Pediatr, 138:441-3.

Cantor LB. 2000. The evolving pharmacotherapeutic profile of brimonidine, an alpha 2-adrenergic agonist, after four years of continuous use. Expert Opin Pharmacother, 1:815-34.

Carrasco Font C, Arias Puente A, Garcia Saenz MC, et al. 2004. [Efficiency of brimonidine $0.2 \%$ and dorzolamide $2 \%$ as adjunctive therapy to beta-blockers]. Arch Soc Esp Oftalmol, 79:163-8.

Choudhri S, Wand M, Shields MB. 2000. A comparison of dorzolamidetimolol combination versus the concomitant drugs. Am JOphthalmol, 130:832-3.

Craven ER, Walters TR, Williams R, et al. 2005. Brimonidine and timolol fixed-combination therapy versus monotherapy: a 3-month randomized trial in patients with glaucoma or ocular hypertension. $J$ Ocul Pharmacol Ther, 21:337-48.

Cvenkel B, Ihan A. 2002. Ocular surface changes induced by topical antiglaucoma monotherapy. Ophthalmologica, 216:175-9.

David R. 2001. Brimonidine (Alphagan): a clinical profile four years after launch. Eur J Ophthalmol, 11(Suppl 2):S72-7.

Donello JE, Padillo EU, Webster ML, et al. 2001. alpha2-Adrenoceptor agonists inhibit vitreal glutamate and aspartate accumulation and preserve retinal function after transient ischemia. $J$ Pharmacol Exp Ther, 296:216-23.

Dong JQ, Babusis DM, Welty DF, et al. 2004. Effects of the preservative purite on the bioavailability of brimonidine in the aqueous humor of rabbits. J Ocul Pharmacol Ther, 20:285-92.

Drance SM. 1997. Glaucoma: a look beyond intraocular pressure. Am J Ophthalmol, 123:817-9.

Fechtner RD, Realini T. 2004. Fixed combinations of topical glaucoma medications. Curr Opin Ophthalmol, 15:132-5.

Ferencz JR, Gilady G, Harel O, et al. 2005. Topical brimonidine reduces collateral damage caused by laser photocoagulation for choroidal neovascularization. Graefes Arch Clin Exp Ophthalmol, 243:877-80.

Gõni FJ; Brimonidine-Timolol Fixed Combination Study Group. 2005. $12-$ week study comparing the fixed combination of brimonidine and timolol with concomitant use of the individual components in patients with glaucoma and ocular hypertension. Eur J Ophthalmol, 15:581-90.
Grant R, Ajello M, Vlass E. 1996. Salt water or high tech? A look at two new rinsing solutions for contact lenses. Optician, 212:38-41.

Hedman K, Alm A. 2000. A pooled-data analysis of three randomized, double-masked, six-month clinical studies comparing the intraocular pressure reducing effect of latanoprost and timolol. Eur J Ophthalmol, 10:95-104

Heijl A, Leske MC, Bengtsson B, et al. 2002. Reduction of intraocular pressure and glaucoma progression: results from the Early Manifest Glaucoma Trial. Arch Ophthalmol, 120:1268-79.

Higginbotham EJ, Schuman JS, Goldberg I, et al. 2002. One-year, randomized study comparing bimatoprost and timolol in glaucoma and ocular hypertension. Arch Ophthalmol, 120:1286-93.

Kass MA, Heuer DK, Higginbotham EJ, et al. 2002. The Ocular Hypertension Treatment Study: a randomized trial determines that topical ocular hypotensive medication delays or prevents the onset of primary open-angle glaucoma. Arch Ophthalmol, 120:701-13.

Katz LJ, Brimonidine Study Group. 1999. Brimonidine tartrate 0.2\% twice daily vs timolol $0.5 \%$ twice daily: 1 -year results in glaucoma patients. Am J Ophthalmol, 127:20-6.

Katz LJ; Brimonidine-Purite Study Groups 1 and 2. 2002. Twelve-month evaluation of brimonidine-purite versus brimonidine in patients with glaucoma or ocular hypertension. J Glaucoma, 11:119-26.

Kent AR, Nussdorf JD, David R, et al. 2001. Vitreous concentration of topically applied brimonidine tartrate $0.2 \%$. Ophthalmology, 108:784-

Klein BE, Klein R, Sponsel WE, et al. 1992. Prevalence of glaucoma. The Beaver Dam Eye Study. Ophthalmology, 99:1499-504.

Konstas AG, Karabatsas CH, Lallos N, et al. 2005. 24-hour intraocular pressures with brimonidine purite versus dorzolamide added to latanoprost in primary open-angle glaucoma subjects. Ophthalmology, 112:603-8

LeBlanc RP. 1998. Twelve-month results of an ongoing randomized trial comparing brimonidine tartrate $0.2 \%$ and timolol $0.5 \%$ given twice daily in patients with glaucoma or ocular hypertension. Ophthalmology, 105:1960-7.

Lee DA, Gornbein J, Abrams C. 2000. The effectiveness and safety of brimonidine as mono-, combination, or replacement therapy for patients with primary open-angle glaucoma or ocular hypertension: a post hoc analysis of an open-label community trial. J Ocul Pharmacol Ther, 16:3-18.

Lee DA, Gornbein JA. 2001. Effectiveness and safety of brimonidine as adjunctive therapy for patients with elevated intraocular pressure in a large, open-label community trial. J Glaucoma, 10:220-6.

Lee DA, Higginbotham EJ. 2005. Glaucoma and its treatment: a review. Am J Health Syst Pharm, 62:691-9.

Leske MC, Heijl A, Hussein M, et al. 2003. Factors for glaucoma progression and the effect of treatment: the early manifest glaucoma trial. Arch Ophthalmol, 121:48-56.

Lichter PR, Musch DC, Gillespie BW, et al. 2001. Interim clinical outcomes in the Collaborative Initial Glaucoma Treatment Study comparing initial treatment randomized to medications or surgery. Ophthalmology, 108:1943-53.

Mayor-Torroglosa S, De la Villa P, Rodriguez ME, et al. 2005. Ischemia results 3 months later in altered ERG, degeneration of inner layers, and deafferented tectum: neuroprotection with brimonidine. Invest Ophthalmol Vis Sci, 46:3825-35.

Melamed S, David R. 2000. Ongoing clinical assessment of the safety profile and efficacy of brimonidine compared with timolol: year-three results. Brimonidine Study Group II. Clin Ther, 22:103-11.

Morrison JC. 2005. Elevated intraocular pressure and optic nerve injury models in the rat. $J$ Glaucoma, 14:315-7.

Netland PA, Landry T, Sullivan EK, et al. 2001. Travoprost compared with latanoprost and timolol in patients with open-angle glaucoma or ocular hypertension. Am J Ophthalmol, 132:472-84.

Netland PA, Michael M, Rosner SA, et al. 2003. Brimonidine Purite and bimatoprost compared with timolol and latanoprost in patients with glaucoma and ocular hypertension. Adv Ther, 20:20-30. 
Noecker R. 2001. Effects of common ophthalmic preservatives on ocular health. Adv Ther, 18:205-15.

Noecker RJ, Herrygers LA, Anwaruddin R. 2004. Corneal and conjunctival changes caused by commonly used glaucoma medications. Cornea, 23:490-6.

Olthoff CM, Schouten JS, van de Borne BW, et al. 2005. Noncompliance with ocular hypotensive treatment in patients with glaucoma or ocular hypertension. An evidence-based review. Ophthalmology, 112:953-61.

Osborne NN, Chidlow G, Layton CJ, et al. 2004. Optic nerve and neuroprotection strategies. Eye, 18:1075-84.

Prok L, Hall D. 2003. A 24-day old with episodic lethargy, hypotonia, and apnea: the eyes have it. Curr Opin Pediatr, 15:226-8.

Rasmussen CA, Kaufman PL. 2005. Primate glaucoma models. $J$ Glaucoma, 14:311-14.

Sall KN, Greff LJ, Johnson-Pratt LR, et al. 2003. Dorzolamide/timolol combination versus concomitant administration of brimonidine and timolol: six-month comparison of efficacy and tolerability. Ophthalmology, 110:615-24.

Schuman JS. 2000. Effects of systemic beta-blocker therapy on the efficacy and safety of topical brimonidine and timolol. Ophthalmology, 107:1171-7.

Schuman JS, Horwitz B, Choplin NT, et al. 1997. A 1-year study of brimonidine twice daily in glaucoma and ocular hypertension. A controlled, randomized, multicenter clinical trial. Arch Ophthalmol, $115: 847-52$

Sharpe ED, Day DG, Beischel CJ, et al. 2004. Brimonidine purite $0.15 \%$ versus dorzolamide $2 \%$ each given twice daily to reduce intraocular pressure in subjects with open angle glaucoma or ocular hypertension. Br J Ophthalmol, 88:953-6.

Simmons ST. 2001. Efficacy of brimonidine $0.2 \%$ and dorzolamide $2 \%$ as adjunctive therapy to beta-blockers in adult patients with glaucoma or ocular hypertension. Clin Ther, 23:604-19.

Simmons ST, Earl ML. 2002. Three-month comparison of brimonidine and latanoprost as adjunctive therapy in glaucoma and ocular hypertension patients uncontrolled on beta-blockers: tolerance and peak intraocular pressure lowering. Ophthalmology, 109:307-14; discussion 14-15.

Skuta GL, Parrish RK, 2nd. 1987. Wound healing in glaucoma filtering surgery. Surv Ophthalmol, 32:149-70.

Sleath B, Robin AL, Covert D, et al. 2006. Patient-reported behavior and problems in using glaucoma medications. Ophthalmology, 113:431-6.

Solish AM, DeLucca PT, Cassel DA, et al. 2004. Dorzolamide/timolol fixed combination versus concomitant administration of brimonidine and timolol in patients with elevated intraocular pressure: a 3-month comparison of efficacy, tolerability, and patient-reported measures. $J$ Glaucoma, 13:149-57.
Stewart WC, Sharpe ED, Harbin TS, Jr., et al. 2000. Brimonidine 0.2\% versus dorzolamide $2 \%$ each given three times daily to reduce intraocular pressure. Am J Ophthalmol, 129:723-7.

Taylor SA, Galbraith SM, Mills RP. 2002. Causes of non-compliance with drug regimens in glaucoma patients: a qualitative study. $J$ Ocul Pharmacol Ther, 18:401-9.

Toris CB, Camras CB, Yablonski ME. 1999. Acute versus chronic effects of brimonidine on aqueous humor dynamics in ocular hypertensive patients. Am J Ophthalmol, 128:8-14.

Toris CB, Gleason ML, Camras CB, et al. 1995. Effects of brimonidine on aqueous humor dynamics in human eyes. Arch Ophthalmol, 113:1514-7.

Tsai JC, Chang HW. 2005. Comparison of the effects of brimonidine $0.2 \%$ and timolol $0.5 \%$ on retinal nerve fiber layer thickness in ocular hypertensive patients: a prospective, unmasked study. $J$ Ocul Pharmacol Ther, 21:475-82.

Vidal-Sanz M, Lafuente MP, Mayor-Torroglosa S, et al. 2001. Brimonidine's neuroprotective effects against transient ischaemiainduced retinal ganglion cell death. Eur J Ophthalmol, 11(Suppl 2):S36-40.

Walters TR. 1996. Development and use of brimonidine in treating acute and chronic elevations of intraocular pressure: a review of safety, efficacy, dose response, and dosing studies. Surv Ophthalmol, 41(Suppl 1):S19-26.

Weinreb RN, Levin LA. 1999. Is neuroprotection a viable therapy for glaucoma? Arch Ophthalmol, 117:1540-4.

Whitson JT, Henry C, Hughes B, et al. 2004. Comparison of the safety and efficacy of dorzolamide $2 \%$ and brimonidine $0.2 \%$ in patients with glaucoma or ocular hypertension. J Glaucoma, 13:168-73.

Wilhelm B, Ludtke H, Wilhelm H. 2006. Efficacy and tolerability of $0.2 \%$ brimonidine tartrate for the treatment of acute non-arteritic anterior ischemic optic neuropathy (NAION): a 3-month, double-masked, randomised, placebo-controlled trial. Graefes Arch Clin Exp Ophthalmol, 244:551-8.

Winfield AJ, Jessiman D, Williams A, et al. 1990. A study of the causes of non-compliance by patients prescribed eyedrops. $\mathrm{Br} J$ Ophthalmol, 74:477-80.

WoldeMussie E, Ruiz G, Wijono M, et al. 2001. Neuroprotection of retinal ganglion cells by brimonidine in rats with laser-induced chronic ocular hypertension. Invest Ophthalmol Vis Sci, 42:2849-55.

Yoles E, Wheeler LA, Schwartz M. 1999. Alpha2-adrenoreceptor agonists are neuroprotective in a rat model of optic nerve degeneration. Invest Ophthalmol Vis Sci, 40:65-73.

Zabriskie N, Netland PA. 2003. Comparison of brimonidine/latanoprost and timolol/dorzolamide: two randomized, double-masked, parallel clinical trials. Adv Ther, 20:92-100. 